\title{
Measuring the Hip Adductor to Abductor Strength Ratio in Ice Hockey and Soccer Players: A Critically Appraised Topic
}

\author{
Robert Rodriguez
}

\begin{abstract}
Clinical Scenario: Ice hockey and soccer are both dynamic sports that involve continuous, unpredictable play. These athletes consistently demonstrate higher rates of groin strains compared with other contact sports. Measuring the hip adductor/ abductor ratio has the potential to expose at-risk players, reduce injury rates, and preserve groin health in players with chronic strains. Focused Clinical Question: What is the clinical utility of measuring the hip adductor/abductor ratio for preseason and in-season ice hockey and soccer players? Summary of Key Findings: Three studies, all of which were prospective cohort designs, were included. One study involved assessing preseason strength and flexibility as a risk factor for adductor strains in professional ice hockey players. Another study performed with the same professional hockey team used preseason hip adductor/abductor strength ratios to screen for those players who would benefit from a strengthening intervention aimed at reducing the incidence of adductor strains. The final study, which was performed in elite U17 soccer players, assessed the effectiveness of monthly in-season strength monitoring as a guide to trigger in-season interventions to decrease injury incidence. Clinical Bottom Line: Measuring the hip adductor/abductor strength ratio in hockey and soccer players can be a beneficial preseason and in-season tool to predict future groin strain risk and screen for athletes who might benefit from a strengthening intervention. Strength of Recommendation: Level 3 evidence exists to support monitoring the hip adductor/abductor strength ratio to assess and reduce the risk of adductor strains in ice hockey and soccer players.
\end{abstract}

Keywords: physical therapy, physiology, rehabilitation, sport medicine, testing and measurement

\section{Clinical Scenario}

Ice hockey and soccer consistently demonstrate higher rates of groin strains compared with other contact sports. Orchard, ${ }^{1}$ in a recent systematic review, concluded that when comparing 15 different college sports, men and women's ice hockey had the highest rates of groin strains $(13.1 \%$ and $12 \%$ of all practice injuries, respectively). Men's soccer had the second highest injury rates (7.8\% of all practice injuries). In elite-level Swedish ice hockey players, Lorentzon et $\mathrm{al}^{2}$ reported that adductor strains accounted for $10 \%$ of all injuries. In the National Hockey League (NHL), Epstein et $\mathrm{al}^{3}$ reported that out of 1441 players from 2006 to 2010, 890 sustained a hip or groin injury. Perhaps the most well understood risk factor for groin strains is reduced hip adductor strength. This includes isolated adductor strength and adductor relative to abductor strength (adductor/abductor ratio). ${ }^{4,5}$ Tyler et $\mathrm{al}^{5}$ reported that NHL players who sustained an adductor strain had on average $18 \%$ lower preseason isolated adductor strength values compared with uninjured players. In addition, the injured players had significantly lower adductor/ abductor strength ratios ( $78 \%$ vs $95 \%$, respectively). Several studies $^{5-7}$ have provided evidence to support measuring the hip adductor/abductor strength ratio throughout the course of a season to identify players who are at risk for sustaining a groin strain.

Rodriguez is with the Ice Hockey Physical Therapy Fellowship Program, Fairview-University of Minnesota Health, Minneapolis, MN. Rodriguez (RobertRodriguez842@gmail.com) is corresponding author.

\section{Focused Clinical Question}

What is the clinical utility of measuring the hip adductor/abductor strength ratio for preseason and in-season ice hockey and soccer players?

\section{Summary of Search, "Best Evidence" Appraised, and Key Findings}

- The literature was searched for any articles that reported measuring the adductor/abductor strength ratio in preseason or in-season hockey and soccer players. Three prospective cohort studies ${ }^{5-7}$ were identified.

- Tyler et $\mathrm{al}^{5}$ performed preseason screening examinations on NHL players, which included hip flexibility, hip strength, and injury history. The authors reported that the hip adductor/abductor strength ratio was the best predictor of a future adductor strain. Relative risk for an adductor strain was $17: 1$ based on a hip adductor to abductor ratio less than $80 \%$.

- Building on their previous study, Tyler et $\mathrm{al}^{6}$ once again performed preseason screening examinations on NHL players. Players with adductor/abductor strength ratios $<80 \%$ participated in a 6 -week $(3 \times / \mathrm{wk})$ preseason exercise program focusing on adductor strengthening (concentric, eccentric, and "functional"). There was no formal control group. Players were followed for 2 consecutive seasons. The authors reported a statistically significant reduction in incidence compared with the previous 2 years with the same team (3 documented adductor strains vs 11 , respectively). 
- Wollin et $\mathrm{al}^{7}$ followed elite male Australian U17 soccer players throughout the course of 2 seasons. Strength measurements, which were recorded during the preseason and monthly during the season, included eccentric adductor strength and the adductor/abductor strength ratio. Alerts were triggered if players demonstrated a $15 \%$ drop in unilateral eccentric hip adductor strength or an adductor/abduction strength ratio $<90 \%$. Triggered players were then retested later that afternoon. If strength measures were not restored, this resulted in time loss from play and remedial-strengthening interventions until their measures were restored. Adductor/abductor strength ratios were lowest at preseason testing and increased significantly by month 2 . The authors proposed that soccer-specific strength can deteriorate after an off-season break and that regular monitoring of strength can help determine a player's readiness to loading.

\section{Clinical Bottom Line}

Level 3 evidence exists to support measuring the hip adductor/ abductor strength ratio in ice hockey and soccer players to screen for risk of sustaining an adductor strain. Level 3 evidence exists to support monitoring and correcting this ratio in-season for soccer players to reduce injury risk and time loss. Level 3 evidence exists to support the use of a preseason or in-season adductor strengthening intervention to reduce risk of injury in ice hockey and soccer players.

\section{Strength of Recommendation}

There is moderate evidence to suggest that measuring the hip adductor/abductor strength ratio in hockey and soccer players can be a beneficial preseason and in-season tool to predict future groin strain risk and screen for athletes who might benefit from a strengthening intervention. This may help ultimately preserve groin health and reduce time loss due to injury. Although the studies reviewed in this critically appraised topic highlight the benefits of measuring and monitoring the hip adductor/abductor strength ratio, each study has limitations.

\section{Search Strategy}

\section{Terms Used to Guide Search Strategy}

- Patient Group: ice hockey and soccer players

- Intervention: measuring the hip adductor/abductor strength ratio

- Comparison: none

- Outcome: groin strain incidence and/or strength improvement

\section{Sources of Evidence and Databases Searched}

- PubMed

- Cochrane

- Google Scholar

- Physiotherapy Evidence Database (PEDro)

\section{Inclusion and Exclusion Criteria}

\section{Inclusion Criteria}

- Preseason and/or in-season hockey and soccer players (all levels)
- Assessment of hip adductor/abductor strength ratio

- Documentation of groin health (via injury records or outcome measures)

- Available in English

\section{Exclusion Criteria}

- Studies that did not follow athletes over the course of at least 1 season

\section{Results of Search}

Three relevant studies ${ }^{5-7}$ were found and are represented in Table 1. All studies were cohort designs.

\section{Best Evidence}

The selected studies were the best match in accordance with the inclusion and exclusion criteria listed above. This represents the highest available evidence that measured hip adductor/abductor strength ratio and collected injury data throughout the course of an entire ice hockey or soccer season. Summaries of the selected study characteristics are presented in Table 2.

\section{Implications for Practice, Education, and Future Research}

Ice hockey and soccer are both dynamic sports that involve repetitive directional changes, body contact, kicking, and striding in an unpredictable environment. This increases the demand on the hips and adductor musculature to control for high acceleration and deceleration forces. ${ }^{8}$ Understanding the physical requirements of each sport can help one appreciate the importance of maintaining a high adductor to abductor strength ratio.

In a recent prospective study involving 110 athletes in various sports, Serner et $\mathrm{al}^{9}$ found that kicking was the most common mechanism for acute groin injuries in soccer. The adductor longus was the most frequently injured muscle, and $81 \%$ of injuries involved the kicking leg. Charnock et $\mathrm{al}^{10}$ performed motion analysis on Division 1 college male soccer players during maximal effort soccer kicking. The authors found that the adductor longus is most susceptible to injury at the end of the windup phase when the kicking leg is in a position of maximum hip extension. At this position, the adductor longus demonstrated both maximum eccentric activation and maximum rate of lengthening. It also represented a transition point from eccentric to concentric activation during initiation of hip flexion. The authors concluded that risk of an adductor longus injury increases with an increase in peak hipextension angle. ${ }^{10}$

\section{Table 1 Summary of Selected Study Designs}

\begin{tabular}{|c|c|c|c|}
\hline $\begin{array}{l}\text { Level of } \\
\text { evidence }\end{array}$ & Study design & $\begin{array}{c}\text { Year } \\
\text { published }\end{array}$ & Reference \\
\hline $2 b$ & Prospective cohort & 2001 & Tyler et $\mathrm{al}^{5}$ \\
\hline $2 b$ & $\begin{array}{l}\text { Prospective cohort, } \\
\text { risk factor prevention }\end{array}$ & 2002 & Tyler et $\mathrm{al}^{6}$ \\
\hline $2 b$ & Longitudinal cohort & 2018 & Wollin et $\mathrm{al}^{7}$ \\
\hline
\end{tabular}




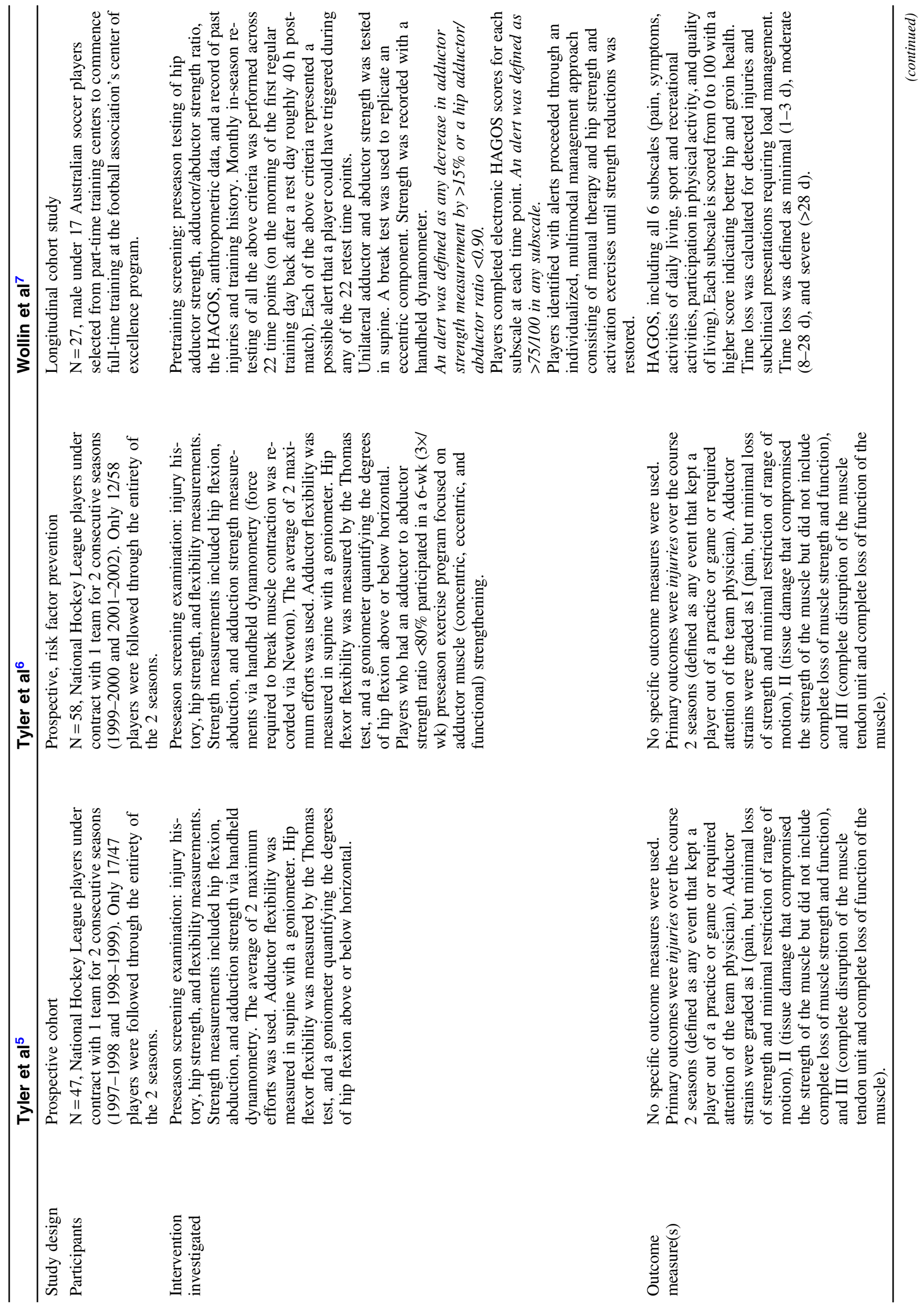




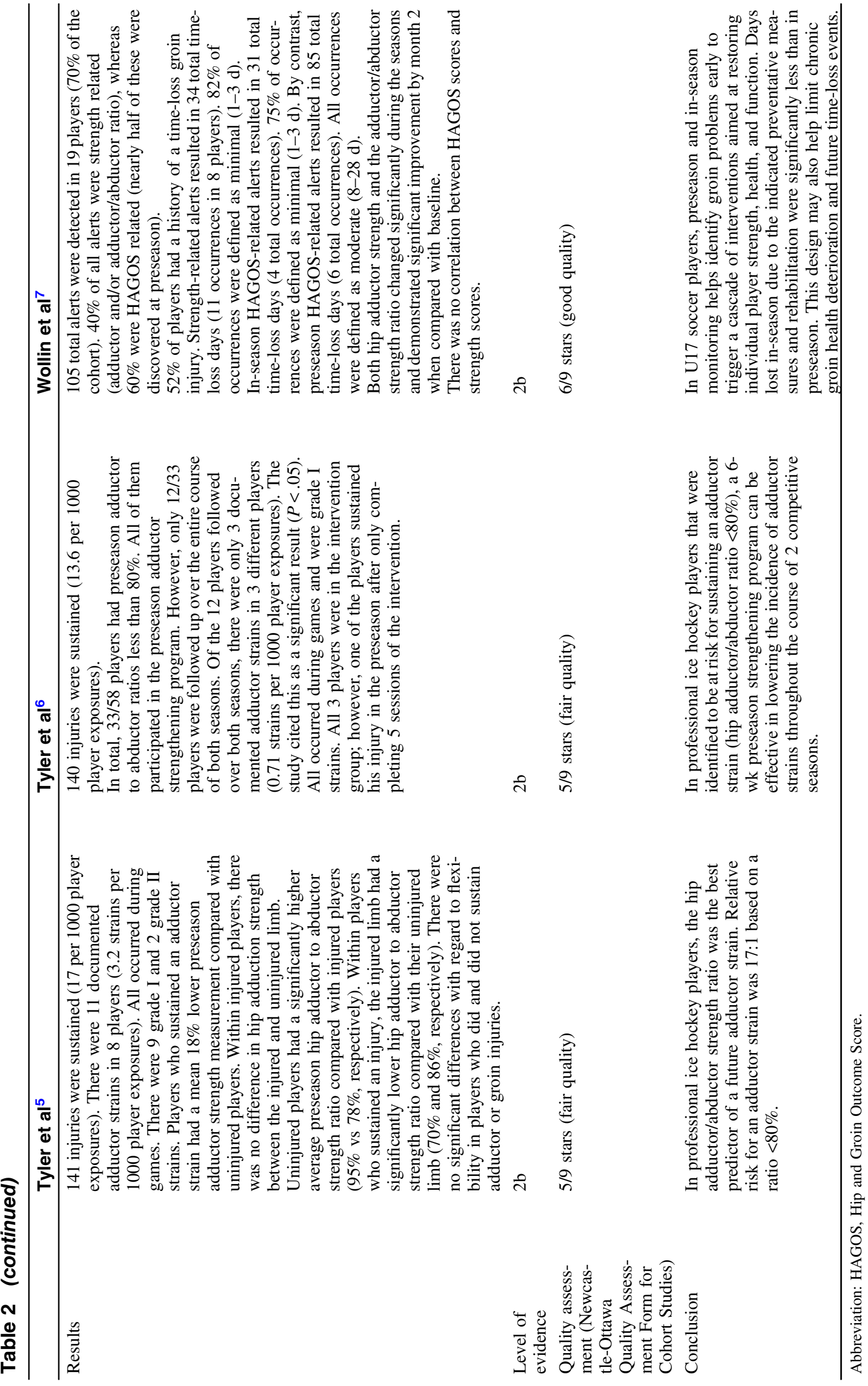


In ice hockey, normal forward striding consists of large propulsive forces produced by the hip extensor and abductor musculature. The stride leg pushes into a position of hip extension, abduction, external rotation, knee extension, and ankle plantar flexion. The adductors act as antagonists to decelerate and control for the explosive abductors. This requires an adequate balance of strength between the adductors and abductors to prevent excessive stride length and risk of adductor strains. At the end of initial stride propulsion, the adductors and hip flexors then quickly transition from working eccentrically to concentrically to reposition the skate under the body for stride turnover. This period of time, immediately after the skate pushes off until it is brought forward to push off again, is defined as the recovery phase. ${ }^{11}$ The recovery phase is especially important in ice hockey. Faster skaters spend less time in this phase and have faster stride turnover, leading to increases in overall skating velocity. ${ }^{11}$ This highlights the need for both eccentric and concentric adductor strength in controlling stride length, improving stride turnover, and reducing risk of injury.

Based on these repetitive mechanisms, it is not surprising that the incidence of adductor strains is higher among ice hockey and soccer athletes compared with those in other contact sports. ${ }^{1}$ These athletes must build and maintain adequate hip adductor strength throughout the course of a season to reduce their risk of injury. So how should clinicians utilize the hip adductor/abductor strength ratio? In addition, what are the implications of monitoring this ratio throughout the course of a season?

When working with ice hockey and soccer players, clinicians should measure the adductor/abductor ratio as a preseason screen to help identify those who are at higher risk for sustaining adductor strains. Tyler et $a{ }^{5}{ }^{5}$ in the first article reviewed in this critically appraised topic, demonstrated the importance of hip adductor strength as a predictor of future adductor strain. Injury risk was 1 per 3.75 hips that tested an adductor/abductor ratio less than $80 \%$, with a relative risk for sustaining an adductor strain of 17:1. With regard to timing of assessment, the preseason appears to have higher vulnerability to injury. Data exist showing that adductor strains were $20 \times$ more frequent during training camp compared with regular training, suggesting that training errors such as preseason deconditioned states and lower levels of sport-specific training play a role ${ }^{4,8}$

The hip adductor/abductor strength ratio should also be used to help clinicians decide which athletes should participate in a strengthening intervention focused on reducing risk of adductor strains. In the second study reviewed in this critically appraised topic, Tyler et $\mathrm{al}^{6}$ concluded that the preseason hip strengthening in professional ice hockey players who had adductor/abductor strength ratios less than $80 \%$ lowered the incidence of adductor strains compared with the previous season. Although this study did demonstrate an effective and statistically significant intervention, it had a few key limitations. Of the 33 players who were identified to have adductor/abductor strength ratios less than $80 \%, 25$ were either cut from the team or had dropped out, subsequently excluding them from the study and any true determination if injury had occurred. In addition, of the players who did sustain a groin strain, all had participated to some extent in the preseason strengthening intervention. Compliance was not reported for these players. Finally, the authors did not retest strength ratios in any players at the completion of the strengthening intervention or throughout the course of the season, which makes it unclear if players had truly restored their ratios above $80 \%$ prior to any reinjury.
In addition to measuring the adductor/abductor strength ratio in the preseason, clinicians should utilize continuous monitoring throughout the course of a season. Wollin et al, ${ }^{7}$ in the third article reviewed in this critically appraised topic, proposed the method of early detection and continuous monthly monitoring of strength ratios as a means of secondary prevention. The authors reported significant improvements in strength ratios from preseason, with the largest increases shown at month 2. This method allowed for players to be flagged and appropriately addressed routinely throughout the entire season. The authors concluded that this method could provide a promising alternative and/or complement to primary prevention efforts in reducing overall time loss due to injury. Considering that athletes with a history of a groin strain are $2.4 \times$ more likely to have recurrence ${ }^{4}$ frequent monitoring may be crucial in preserving long-term groin health.

Groins strains cannot absolutely be prevented, but proper screening and applied strengthening interventions can help significantly reduce an athlete's risk of injury. There is good clinical utility for measuring the hip adductor/abductor strength ratio both at preseason and continuously throughout the course of a season. The ratio should be used as a screen for injury risk and as a funnel for those who would benefit from active strengthening interventions in hopes of reducing overall incidence.

\section{Acknowledgment}

I would like to acknowledge and thank David A. Krause, PT, D.S.c, OCS and John H. Hollman, Ph.D. at Mayo Clinic in Rochester, MN for their contributions.

\section{References}

1. Orchard JW. Men at higher risk of groin injuries in elite team sports: a systematic review. Br J Sports Med. 2015;49:798-802. PubMed ID: 26031645 doi:10.1136/bjsports-2014-094272

2. Lorentzon $R$, Wedrèn $H$, Pietilä $T$. Incidences, nature, and causes of ice hockey injuries: a three year prospective study of a Swedish elite ice hockey team. Am J Sports Med. 1998;16(4):392-396. doi:10. 1177/036354658801600415

3. Epstein DM, McHugh M, Yorio M, Neri B. Intra-articular hip injuries in National Hockey League players: a descriptive epidemiological study. Am J Sports Med. 2013;41(2):343-348. PubMed ID: 23193146 doi:10.1177/0363546512467612

4. Whittaker JL, Small C, Maffey L, Emery CA. Risk factors for groin injury in sport: an updated systematic review. Br J Sports Med. 2015; 49:803-809. PubMed ID: 25833903 doi:10.1136/bjsports-2014094287

5. Tyler T, Nicholas SJ, Campbell RJ, McHugh MP. The association of hip strength and flexibility with the incidence of adductor muscle strains in professional ice hockey players. Am J Sports Med. 2001;29(2):124-128. PubMed ID: 11292035 doi:10.1177/ 03635465010290020301

6. Tyler T, Nicholas SJ, Campbell RJ, Donellan S, McHugh MP. The effectiveness of a preseason exercise program to prevent adductor muscle strains in professional ice hockey players. Am J Sports Med. 2002;30(5):680-683. PubMed ID: 12239001 doi:10.1177/ 03635465020300050801

7. Wollin M, Thorborg K, Welvaert M, Pizzari T. In-season monitoring of hip and groin strength, health and function in elite youth soccer: implementing an early detection and management strategy over two consecutive seasons. J Sci Med Sport. 2018;21(10):988-993. PubMed ID: 29555148 doi:10.1016/j.jsams.2018.03.004 
8. Sedaghati P, Alizadeh MH, Shirzad E, Ardjmand A. Review of sportinduced groin injuries. Trauma Mon. 2013;18(3):107-112. PubMed ID: 24350166 doi:10.5812/traumamon.12666

9. Serner A, Tol JL, Jomaah N, et al. Diagnosis of acute groin injuries: a prospective study of 110 athletes. Am J Sports Med. 2015;43(8): 1857-1864. PubMed ID: 25977522 doi:10.1177/0363546515585123
10. Charnock BL, Lewis CL, Garrett WE Jr, Queen RM. Adductor longus mechanics during the maximal effort soccer kick. Sports Biomech. 2009;8(3):223-234. PubMed ID: 19891200 doi:10.1080/ 14763140903229500

11. Bracko MR. Biomechanics powers ice hockey performance. Sports Med. 2004;47-53. 